\title{
ON THE PHYSICAL ORIGIN OF THE OPPENHEIMER-AHLUWALIA ZERO-ENERGY SOLUTIONS OF MAXWELL EQUATIONS
}

\author{
ANDREW E. CHUBYKALO \\ Escuela de Física, Universidad Autónoma de Zacatecas \\ Apartado Postal C-580 Zacatecas 98068, ZAC., México
}

\begin{abstract}
In virtue of the Chubykalo - Smirnov-Rueda generalized form of the Maxwell-Lorentz equation a new form of the energy density of the electromagnetic field was obtained. This result allows us to explain a physical origin of the Oppenheimer-Ahluwalia zero-energy solutions of the Maxwell equations.
\end{abstract}

\section{Introduction}

If $\phi_{L}(\mathbf{p})$ and $\phi_{R}(\mathbf{p})$ represent the massless $(1,0)$ and $(0,1)$ fields respectively [1], then the source-free momentum-space Maxwell equation can be written as (see, e.g., Ref. [2] $)^{\mathrm{a}}$

$$
\begin{aligned}
& \left(\mathbf{J} \cdot \mathbf{p}+p^{0}\right) \phi_{L}(\mathbf{p})=0 \\
& \left(\mathbf{J} \cdot \mathbf{p}-p^{0}\right) \phi_{R}(\mathbf{p})=0
\end{aligned}
$$

where $\mathbf{J}$ are the $3 \times 3$ spin- 1 angular momentum matrices

$$
J_{x}=\left(\begin{array}{ccc}
0 & 0 & 0 \\
0 & 0 & -i \\
0 & i & 0
\end{array}\right), \quad J_{y}=\left(\begin{array}{ccc}
0 & 0 & i \\
0 & 0 & 0 \\
-i & 0 & 0
\end{array}\right), \quad J_{z}=\left(\begin{array}{ccc}
0 & -i & 0 \\
i & 0 & 0 \\
0 & 0 & 0
\end{array}\right) .
$$

Oppenheimer [3] and Ahluwalia [2, 4.5 independently noted that in order that nontrivial solutions of Eqs. (1) and (2) exist one must have

$$
p^{0}= \pm|\mathbf{p}|, \quad p^{0}=0 .
$$

aThe configuration-space Maxwell equations follow on setting

$$
\mathbf{p}=-i \nabla, \quad p^{0}=i \frac{\partial}{\partial t},
$$

and making appropriate linear combinations of the $\phi_{R}(x)$ and $\phi_{L}(x)$ to obtain the $\mathbf{E}$ and $\mathbf{H}$ fields. 
These "dispersion relations" follow from the condition Det. $\left(\mathbf{J} \cdot \mathbf{p} \pm p^{0}\right)=0$.

This situation immediately raises two problems: $(i)$ there are negative energy solutions, and (ii) the equations support solutions with zero energy. One may either declare that the negative energy solutions, and solutions with identically vanishing energy content, are to be discarded. Or, face the possibility that the usual "quadratic in $\mathbf{E}$ and $\mathbf{H}$ " expression for the energy density of the electromagnetic field is not complete. Here, I argue that the latter is the case by providing an explicit construct for such an indicated modified expression for the energy density.

Let us recall a generally accepted way to obtain the energy density of the electromagnetic field in vacuum [6].

In order to obtain the energy density of the electromagnetic field and the density of the flux of the electromagnetic energy Landau (see $\S 34$, p.76 in [6]) uses two of Maxwell's equations:

$$
\nabla \times \mathbf{H}=\frac{4 \pi}{c} \mathbf{j}+\frac{1}{c} \frac{\partial \mathbf{E}}{\partial t}
$$

and

$$
\nabla \times \mathbf{E}=-\frac{1}{c} \frac{\partial \mathbf{H}}{\partial t}
$$

Landau multiplies both sides of (5) by $\mathbf{E}$ and both sides of (6) by $\mathbf{H}$ and combines the resultant equations:

$$
\frac{1}{c} \mathbf{E} \cdot \frac{\partial \mathbf{E}}{\partial t}+\frac{1}{c} \mathbf{H} \cdot \frac{\partial \mathbf{H}}{\partial t}=-\frac{4 \pi}{c} \mathbf{j} \cdot \mathbf{E}-(\mathbf{H} \cdot[\nabla \times \mathbf{E}]-\mathbf{E} \cdot[\nabla \times \mathbf{H}])
$$

Then, using the well-known formula of vectorial analysis, one obtains:

$$
\frac{\partial}{\partial t}\left(\frac{E^{2}+H^{2}}{8 \pi}\right)=-\mathbf{j} \cdot \mathbf{E}-\nabla \cdot \mathbf{S}
$$

where the vector

$$
\mathbf{S}=\frac{c}{4 \pi}[\mathbf{E} \times \mathbf{H}]
$$

is called the Poynting vector.

Then Landau integrates (8) over a volume and applies Gauss' theorem to the second term on rhs:

$$
\frac{\partial}{\partial t} \int \frac{E^{2}+H^{2}}{8 \pi} d \mathcal{V}=-\int \mathbf{j} \cdot \mathbf{E} d \mathcal{V}-\oint \mathbf{S} \cdot d \mathbf{f} .
$$

If the integral, Landau writes further, extends over all space, then the surface integral vanishes (the field is zero at infinity). Then one can express the integral $\int \mathbf{j} \cdot \mathbf{E} d \mathcal{V}$ as a sum $\sum q \mathbf{v} \cdot \mathbf{E}$ over all the charges, and substitute from Eq.(17.7, [6]):

$$
q \mathbf{v} \cdot \mathbf{E}=\frac{d}{d t} \mathcal{E}_{\text {kin }}
$$


As a result Landau obtains:

$$
\frac{d}{d t}\left\{\int \frac{E^{2}+H^{2}}{8 \pi} d \mathcal{V}+\sum \mathcal{E}_{\text {kin }}\right\}=0 .
$$

Thus, Landau concludes, for the closed system consisting of the electromagnetic field and particles present in it, the quantity in brackets in this equation is conserved. The second term in this expression is the kinetic energy (including the rest energy of all particles, of course), the first term is consequently the energy of the field it itself. One can therefore call the quantity

$$
w=\frac{E^{2}+H^{2}}{8 \pi}
$$

the energy density of the electromagnetic field. Obviously that it is impossible to coordinate such a definition of the energy density with such a configuration of the fields when $w$ is zero in some point while the fields $\mathbf{E}$ and $\mathbf{H}$ are not zero at the same point.

Here however, we have to make two important comments:

1) Landau uses the transition $\frac{\partial}{\partial t} \int(\ldots) \rightarrow \frac{d}{d t} \int(\ldots)$ for a field too freely, without any clarification of this mathematical operation.

2) Landau states (see [6], $\S 31$ ) that the surface integral $\oint \mathbf{S} \cdot d \mathbf{f}$ vanishes at infinity because the field is zero at infinity. But in this case one implicitly neglects a radiation field which can go off to infinity. In other words, one cannot do the transition from (10) to (11) without imposing certain additional conditions, which prevent this radiation field from going off to infinity. To be more specific, let us turn to Landau ([6], §34, first footnote): "Here we also assume that the electromagnetic field of the system also vanishes at infinity. This means that, if there is a radiation of electromagnetic waves by the system, it is assumed that special 'reflecting walls' prevent these waves from going off to infinity."

Let us, now turn to our (and Landau's) formulas (10) and (11):

In classical electrodynamics one assumes that the energy conservation law is an absolute law and in order to satisfy this law we must, in general, take into account a possible change of energy of these "reflecting walls", which may take place as a result of the energy exchange between these "walls" and the system "particles + fields".

But we cannot know a mathematically correct way to take into account this energy in the formula (11) without exact knowledge of the "nature" of the "reflecting walls." In this case we cannot obtain an exact energy conservation law using the concept of the "reflecting walls." In other words in order to obtain the exact energy conservation law one should not introduce these "walls," but rather we must assume that the surface integral $\oint \mathbf{S} \cdot d \mathbf{f}$ does not vanish at infinity. But in this case Eq.(10) turns into a trivial equality, which although satisfying the exact energy conservation 
law, cannot be used to derive any conclusion about the concrete mathematical form of a energy density of the electromagnetic field.

\section{Another form of energy density and its connection with the Oppenheimer-} Ahluwalia zero-energy solutions of the Maxwell equations

However, there is a way to obtain the explicit form of the energy density of the electromagnetic field. Let me turn to our (with R.Smirnov-Rueda) papers [7,8] where we prove that the electromagnetic field has to be represented by two independent parts:

$$
\begin{aligned}
& \mathbf{E}=\mathbf{E}_{0}+\mathbf{E}^{*}=\mathbf{E}_{0}\left(\mathbf{r}-\mathbf{r}_{q}(t)\right)+\mathbf{E}^{*}(\mathbf{r}, t), \\
& \mathbf{H}=\mathbf{H}_{0}+\mathbf{H}^{*}=\mathbf{H}_{0}\left(\mathbf{r}-\mathbf{r}_{q}(t)\right)+\mathbf{H}^{*}(\mathbf{r}, t) .
\end{aligned}
$$

Here we note that quasistatic components such as $\mathbf{E}_{0}$ and $\mathbf{H}_{0}$ depend only on the distance between the point of observation and the source position at the instant of observation, whereas time-varying-fields such as $\mathbf{E}^{*}$ and $\mathbf{H}^{*}$ depend explicitly on the point of observation and the time of observation.

Let us now rewrite Eqs. (5) and (6) as formulas (45) and (46) from our aforementioned paper [8]:

$$
\begin{aligned}
& \nabla \times \mathbf{H}=\frac{4 \pi}{c} \mathbf{j}+\frac{1}{c} \frac{d \mathbf{E}}{d t} \\
& \nabla \times \mathbf{E}=-\frac{1}{c} \frac{d \mathbf{H}}{d t}
\end{aligned}
$$

where the total time derivative of any vector field value $\mathbf{E}$ (or $\mathbf{H}$ ) can be calculated by the following rule:

$$
\frac{d \mathbf{E}}{d t}=\frac{\partial \mathbf{E}^{*}}{\partial t}-\left(\sum_{i} \mathbf{V}_{i} \cdot \nabla\right) \mathbf{E}_{0}
$$

here $\mathbf{V}_{i}$ are velocities of the particles at the same instant of observation. ${ }^{\text {b }}$

The mutual independence of the fields \{\}$_{0}$ and \{\}$^{*}$ allows us also to rewrite Eqs. (15) and (16) (taking into account relations (13), (14) and (17)) as two uncoupled pairs of differential equations:

$$
\begin{aligned}
& \nabla \times \mathbf{H}^{*}=\frac{1}{c} \frac{\partial \mathbf{E}^{*}}{\partial t}, \\
& \nabla \times \mathbf{E}^{*}=-\frac{1}{c} \frac{\partial \mathbf{B}^{*}}{\partial t}
\end{aligned}
$$

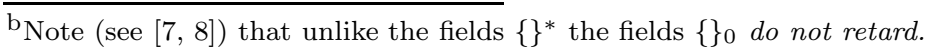


and

$$
\begin{aligned}
& \nabla \times \mathbf{H}_{0}=\frac{4 \pi}{c} \mathbf{j}-\frac{1}{c}\left(\sum_{i} \mathbf{V}_{i} \cdot \nabla\right) \mathbf{E}_{0}, \\
& \nabla \times \mathbf{E}_{0}=\frac{1}{c}\left(\sum_{i} \mathbf{V}_{i} \cdot \nabla\right) \mathbf{H}_{0} .
\end{aligned}
$$

Let us, at last, repeat the calculation of Landau (see above), but now, taking into account Eqs. (15) and (16) and without imposing the "reflecting walls" type condition.

Let us multiply both sides of (15) by $\mathbf{E}$ and both sides of (16) by $\mathbf{H}$ and combine the resultant equations. Then we get:

$$
\frac{1}{c} \mathbf{E} \cdot \frac{d \mathbf{E}}{d t}+\frac{1}{c} \mathbf{H} \cdot \frac{d \mathbf{H}}{d t}=-\frac{4 \pi}{c} \mathbf{j} \cdot \mathbf{E}-(\mathbf{H} \cdot[\nabla \times \mathbf{E}]-\mathbf{E} \cdot[\nabla \times \mathbf{H}]) .
$$

Using the rule (17) and the well-known formula of vector analysis, we obtain:

$$
\begin{aligned}
\frac{1}{c} \mathbf{E} \cdot\left\{\frac{\partial \mathbf{E}^{*}}{\partial t}-\left(\sum_{i} \mathbf{V}_{i} \cdot \nabla\right) \mathbf{E}_{0}\right\}+ & \frac{1}{c} \mathbf{H} \cdot\left\{\frac{\partial \mathbf{H}^{*}}{\partial t}-\left(\sum_{i} \mathbf{V}_{i} \cdot \nabla\right) \mathbf{H}_{0}\right\}= \\
= & -\frac{4 \pi}{c} \mathbf{j} \cdot \mathbf{E}-\nabla \cdot[\mathbf{E} \times \mathbf{H}] .
\end{aligned}
$$

Then, taking into account the relations (13), (14), and also that

$$
\frac{d\{\}^{*}}{d t}=\frac{\partial\{\}^{*}}{\partial t} \quad \text { and } \quad \frac{d\{\}_{0}}{d t}=-\left(\sum_{i} \mathbf{V}_{i} \cdot \nabla\right)\{\}_{0}
$$

and finally, after some transformations we obtain:

$$
\begin{aligned}
\frac{\partial}{\partial t}\left(\frac{E^{* 2}+H^{* 2}}{8 \pi}\right)+ & \frac{d}{d t}\left(\frac{2 \mathbf{E}^{*} \cdot \mathbf{E}_{0}+2 \mathbf{H}^{*} \cdot \mathbf{H}_{0}+E_{0}^{2}+H_{0}^{2}}{8 \pi}\right)= \\
& =-\mathbf{j} \cdot \mathbf{E}-\nabla \cdot\left(\frac{c}{4 \pi}[\mathbf{E} \times \mathbf{H}]\right) .
\end{aligned}
$$

Now we can integrate this expression over a volume (taking into account the relation $\left.q \mathbf{v} \cdot \mathbf{E}=\frac{d}{d t} \mathcal{E}_{\text {kin }}\right):$

$$
\begin{aligned}
\frac{\partial}{\partial t} \int \frac{E^{* 2}+H^{* 2}}{8 \pi} d \mathcal{V}+ & \frac{d}{d t}\left(\int \frac{2 \mathbf{E}^{*} \cdot \mathbf{E}_{0}+2 \mathbf{H}^{*} \cdot \mathbf{H}_{0}+E_{0}^{2}+H_{0}^{2}}{8 \pi} d \mathcal{V}+\sum \mathcal{E}_{\text {kin }}\right)= \\
& =-\int \nabla \cdot\left(\frac{c}{4 \pi}[\mathbf{E} \times \mathbf{H}]\right) d \mathcal{V} .
\end{aligned}
$$

Let us now extend these integrals over all space and apply Gauss' theorem to rhs of (25). In this case, taking into account that the fields \{\}$_{0}$ connected with particles vanish at infinity, we obtain:

$$
-\int \nabla \cdot\left(\frac{c}{4 \pi}[\mathbf{E} \times \mathbf{H}]\right) d \mathcal{V} \rightarrow-\oint\left(\frac{c}{4 \pi}\left[\mathbf{E}^{*} \times \mathbf{H}^{*}\right]\right) \cdot d \mathbf{f}=-\int \nabla \cdot\left(\frac{c}{4 \pi}\left[\mathbf{E}^{*} \times \mathbf{H}^{*}\right]\right) d \mathcal{V}
$$


Andrew E. Chubykalo

It is easy to verify, taking into account Eqs. (18) and (19), that the last integral in (26) and the first integral in (25) are equal to each other. Then (25) becomes:

$$
\frac{d}{d t}\left(\int \frac{2 \mathbf{E}^{*} \cdot \mathbf{E}_{0}+2 \mathbf{H}^{*} \cdot \mathbf{H}_{0}+E_{0}^{2}+H_{0}^{2}}{8 \pi} d \mathcal{V}+\sum \mathcal{E}_{\text {kin }}\right)=0 .
$$

We can therefore call the quantity

$$
w=\frac{2 \mathbf{E}^{*} \cdot \mathbf{E}_{0}+2 \mathbf{H}^{*} \cdot \mathbf{H}_{0}+E_{0}^{2}+H_{0}^{2}}{8 \pi}
$$

the energy density of the electromagnetic field.

Note again that one never can obtain the so called "Oppenheimer-Ahluwalia zero energy solutions" from the generally accepted form of the electromagnetic energy

$$
w=\frac{E^{2}+H^{2}}{8 \pi}
$$

because for real fields this quantity is always positive and only can be zero if the fields $\mathbf{E}$ and $\mathbf{H}$ are zero simultaneously.

But from our new representation of the density of this energy

$$
w=\frac{2 \mathbf{E}^{*} \cdot \mathbf{E}_{0}+2 \mathbf{H}^{*} \cdot \mathbf{H}_{0}+E_{0}^{2}+H_{0}^{2}}{8}
$$

it easy to see that the fields \{\}$^{*}$ and \{\}$_{0}$ can have mutually different signs because these fields \{\}$^{*}$ and \{\}$_{0}$ are different fields. It means that we can have the following relation:

$$
2 \mathbf{E}^{*} \cdot \mathbf{E}_{0}+2 \mathbf{H}^{*} \cdot \mathbf{H}_{0}<0
$$

and, in turn, we can have a configuration of non-zero fields for which $w$ is zero:

$$
2 \mathbf{E}^{*} \cdot \mathbf{E}_{0}+2 \mathbf{H}^{*} \cdot \mathbf{H}_{0}=-\left(E_{0}^{2}+H_{0}^{2}\right)
$$

Actually, it is sufficient that the fields \{\}$^{*}$ and \{\}$_{0}$ satisfy the equations:

$$
\left|\mathbf{E}^{*}\right|=\frac{-\left|\mathbf{E}_{0}\right|}{2 \cos \alpha} \quad \text { and } \quad\left|\mathbf{H}^{*}\right|=\frac{-\left|\mathbf{H}_{0}\right|}{2 \cos \alpha},
$$

where $\alpha$ is an angle between the vectors \{\}$^{*}$ and \{\}$_{0}$ with the following limits:

$$
\frac{\pi}{2}<\alpha<\pi+\frac{\pi}{2} .
$$

From the formulas (30) and (31) one also can see that there are negative energy solutions (compare with the remark (i) after Eq.(4)). 


\section{Conclusion}

In this short note I do not deal with such concepts as the momentum and the angular momentum of the electromagnetic field. And although in this work I use the concept of the Poynting vector, I do not use the concept of the density of momentum of the field. Let me clarify my point of view:

On the one hand, from generally accepted classical electrodynamics we know that the Poynting vector is proportional to the density of the electromagnetic field momentum. But on the other hand, paradoxes connected with the Poynting vector exist and they are well-known. For example, in our paper [7]: if a charge $Q$ is vibrating in some mechanical way along the $X$-axis, then the value of $w$ (which is a point function like $|\mathbf{E}|$ ) on the same axis will be also oscillating. The question arises: how does the test charge $q$ at the point of observation, lying at some fixed distance from the charge $Q$ along the continuation of the $X$-axis, "know" about the charge $Q$ vibration? In other words we have a rather strange situation: the Poynting vector $\mathbf{S}=\frac{c}{4 \pi}[\mathbf{E} \times \mathbf{H}]$ is zero along this axis (because $\mathbf{H}$ is zero along this line) but the energy and the momentum, obviously "pass" from point to point along this axis. This means that we cannot be sure whether using the new definition of the energy density will permit use of the old definition of the momentum density. This problem, I think, requires very careful research. Other quantities of classical electrodynamics such as electromagnetic field tensor, electromagnetic energy-momentum tensor etc. can (and perhaps must) also change their physical meanings. In fact, a considerable number of works have recently been published which directly declare: classical electrodynamics must be very sufficiently reconsidered ${ }^{\mathrm{c}}$. To be more specific, let me end this paper with the words of R.Feynman who wrote [10]: “...this tremendous edifice (classical electrodynamics), which is such a beautiful success in explaining so many phenomena, ultimately falls on its face. When you follow any of our physics too far, you find that it always gets into some kind of trouble. ...the failure of the classical electromagnetic theory. ...Classical mechanics is a mathematically consistent theory; it just doesn't agree with experience. It is interesting, though, that the classical theory of electromagnetism is an unsatisfactory theory all by itself. There are difficulties associated with the ideas of Maxwell's theory which are not solved by and not directly associated with quantum mechanics..."

\section{Acknowledgments}

I am grateful to Dr. D.V.Ahluwalia for many stimulating discussions and critical

cSee a brilliant review of these works "Essay on Non-Maxwellian Theories of Electromagnetism" by V.V.Dvoeglazov [9]. 
comments. I acknowledge the brilliant review by Professor V.Dvoeglazov, which put an idea into me to make the present work. I am grateful to Zacatecas University for a professorship.

\section{References}

1. D. V. Ahluwalia, M. B. Johnson, and T. Goldman, Phys. Lett. B, 316, 102 (1993).

2. D. V. Ahluwalia, Texas A\&M Ph.D thesis (1991); abstracted in Dissertation Abstracts International B 52, 4792-B (1992).

3. J. R. Oppenheimer, Phys. Rev. 38, 725 (1931).

4. D. V. Ahluwalia and D. J. Ernst, Mod. Phys. Lett. A 7, 1967 (1992).

5. D. V. Ahluwalia, in Proseeding of "The Present Status of Quantum Theory of Light: A Simposium to Honour Jean-Pierre Vigier." York University, Toronto, August 27-30, 1995, eds. G. Hunter et al., (Kluwer Academic Publishers, Dordrecht, 1996).

6. Landau L.D and Lifshitz E.M., Teoria Polia (Nauka, Moscow, 1973) [English translation: The Classical Theory of Field (Pergamon, Oxford, 1975)].

7. A. E. Chubykalo and R. Smirnov-Rueda, Phys. Rev. E 535373 (1996); see also the Errata Phys. Rev. E 553793 (1997); and the discussion: Ivezić T. and Škovrlj L., Phys. Rev. E 57(2) (1998); A. E. Chubykalo and R. Smirnov-Rueda, Phys. Rev. E 57(2) (1998).

8. A. E. Chubykalo and R. Smirnov-Rueda, Mod. Phys. Lett. A 12(1), 1 (1997).

9. V. V. Dvoeglazov, Hadronic J. Suppl. 12, 241 (1997).

10. R. P. Feynman, Lectures on Physics: Mainly Electromagnetism and Matter (AddisonWesley, London, 1964). 